\title{
A Mutant of the Bck1 Homolog from Cryphonectria parasitica Resulted in Sectorization with an Impaired Pathogenicity
}

\author{
Jung-Mi Kim, ${ }^{1}$ Joong-Gi Lee, ${ }^{2}$ Suk-Hyun Yun, ${ }^{2}$ Kum-Kang So, ${ }^{2}$ Yo-Han Ko, ${ }^{2}$ Young Ho Kim, ${ }^{3}$ \\ Seung-Moon Park, ${ }^{2}$ and Dae-Hyuk Kim ${ }^{2}$ \\ ${ }^{1}$ Department of Bio-Environmental Chemistry, Institute of Life Science and Natural Resources, Wonkwang University, Iksan, \\ Chonbuk 570-749, Korea; ${ }^{2}$ Institute for Molecular Biology and Genetics, Center for Fungal Pathogenesis, Department of \\ Bioactive Material Sciences, Chonbuk National University, Jeonju, Chonbuk 561-756, Korea; and ${ }^{3}$ Department of Agricultural \\ Biotechnology, Seoul National University, Seoul 151-921, Korea
}

Submitted 20 August 2015. Accepted 31 December 2015.

\begin{abstract}
CpBck1, an ortholog of the cell-wall integrity mitogen-activated protein kinase kinase kinase of Saccharomyces cerevisiae, was cloned and characterized from the chestnut blight fungus Cryphonectria parasitica. The CpBck1-null mutant displayed cell wall integrity-related phenotypic changes such as abnormal cell morphology and wall formation and hypersensitivity to cell wall-disrupting agents. In addition, the mutant showed severely retarded growth without any sign of normal development, such as hyphal differentiation, conidiation, or pigmentation. As the culture proceeded, the mutant colony showed sporadic sectorization. Once sectored, the sectored phenotype of robust mycelial growth without differentiation was stably inherited. Compared with the wild type, both the parental $C p B c k 1$-null mutant and the sectored progeny exhibited marked impaired virulence. The present study revealed that a mutation in a signaling pathway component related to cell-wall integrity resulted in sporadic sectorization and these sectored phenotypes were stably inherited, suggesting that this signal transduction pathway is implicated in adaptive genetic changes for sectorization.
\end{abstract}

Cryphonectria parasitica (Murrill) Barr, the causal agent of chestnut blight, devastated the chestnut forests in North America at the beginning of the 20th century (Van Alfen 1982). However, strains containing single-stranded (ss)RNA Cryphonectria Hypovirus 1 (CHV1) exhibit characteristic symptoms of decreased virulence, a phenomenon referred to as hypovirulence (Anagnostakis 1982; Nuss 1992; Van Alfen et al. 1975), and diverse hypovirulenceassociated phenotypic changes, such as reduced sporulation, female fertility, pigmentation, laccase production, and oxalate accumulation (Elliston 1985; Havir and Anagnostakis 1983; Rigling et al. 1989). The molecular basis of these phenotypic changes led to alterations in host transcriptional profiles in response to the hypovirus infection (Allen and Nuss 2004; Allen et al. 2003; Deng et al. 2007; Kang et al. 1999; Kazmierczak et al. 1996). Considering the array of phenotypic changes in the hypovirulent strain, implementation of a signal transduction

Corresponding author: D.-H. Kim; Telephone: +82-63-270-3440; Fax: +82-63-270-4312; E-mail: dhkim@jbnu.ac.kr

*The $\boldsymbol{e}$-Xtra logo stands for "electronic extra" and indicates that five supplementary figures are published online.

() 2016 The American Phytopathological Society pathway during viral symptom development may be a coordinate and specific mechanism of virus-mediated fungal gene regulation. Accordingly, several fungal signal transduction pathways are perturbed by the hypovirus infection (Chen et al. 1996; Gao and Nuss 1996; Kasahara and Nuss 1997; Kim et al. 2002; Park et al. 2004, 2012; Rostagno et al. 2010).

The mitogen-activated protein kinase (MAPK) signaling pathway was of interest because it is required for numerous processes related to growth, differentiation, and pathogenesis (Xu 2000; Zhao et al. 2007). The MAPK signal transduction pathway is utilized by eukaryotic cells to transduce a wide variety of cellular signals through a stepwise phosphorylation relay. This cascade, which is conserved across a wide variety of organisms from yeast to humans (Herskowitz 1995; Schaeffer and Weber 1999), consists of three functionally interlinked protein kinases: MAPKKK, MAPKK, and MAPK. Although five different MAPK pathways have been identified in Saccharomyces cerevisiae, three MAPK homologs representing the pheromone response pathway (Fus3/Kss1), cell wall-integrity pathway (Slt2), and osmoregulation/stress response pathway (Hog1) are generally found in other higher fungi (Xu 2000). In C. parasitica, several genes in MAPK pathways and their downstream effectors are known to be regulated by the presence of hypovirus or are involved in pathogenicity (Choi et al. 2005; Deng et al. 2007; Park et al. 2004, 2012; Sun et al. 2009; Turina et al. 2006). Among these, the MAPK pathway, which is involved in the cell wall-integrity pathway, has aroused interest because orthologs of yeast Slt2 in plant and human pathogens are important for pathogenicity (Zhao et al. 2007). Furthermore, a previous study using a specific antibody suggested that phosphorylation of Cpkk1, an ortholog of yeast Mkk2 (a cell wall-5integrity MAPKK), was affected by the presence of the hypovirus and was important for virulence in the chestnut tree (Moretti et al. 2014; Turina et al. 2006). However, no other components of the cell wall-integrity MAPK pathway have been examined. In addition, neither the biological function nor downstream effectors in the cell wall-integrity MAPK pathway have been characterized at the molecular level.

In this study, we cloned and characterized $C p B c k l$, an ortholog of yeast $B c k 1$ (a cell wall-integrity MAPKKK), from C. parasitica. Our results showed that, in addition to cell wall-associated phenotypic changes, the loss-of-function mutant of $C p B c k 1$ resulted in sporadic occurrence of sectors, suggesting that a specific MAPK signaling pathway plays a role in sectorization, one of the representative fungal degenerative processes. 


\section{RESULTS}

\section{Database description.}

A MAPKKK gene, $C p B c k 1$, which showed high sequence similarity to known fungal $B c k l$-like genes, was identified in the genome of $C$. parasitica by inspecting the draft genome sequence (MycoCosm database Cryphonectria parasitica archive). polymearse chain reaction (PCR) amplification of the $C p B c k l$ gene, which showed high similarity to other MAPKKKs in the cell wall-integrity pathway, resulted in an expected 850-bp fragment. Southern analysis of total genomic DNA under low-stringency conditions, using the cloned PCR amplicon as a probe, suggested that only a single copy of the cloned gene was present in the $C$. parasitica genome. Based on genomic sequence analysis, a near full-length cDNA clone was obtained using reverse transcription (RT)-PCR with the primer pair $C p B c k 1-c F 1$ and $C p B c k 1$-cR1 at nucleotide positions (nt) -11 to 7 and 5,316 to 5,333 (relative to the start codon), respectively, and the resulting 4.9-kb amplicon was cloned into a pGEM-T Easy vector. A comparison with the corresponding genomic sequences revealed that the cloned gene consisted of four exons, with three intervening sequences of 93, 270, and $108 \mathrm{bp}$. The deduced CpBck1 protein product (CpBCK1) consisted of 1,619 amino acids, with an estimated molecular mass of $173.9 \mathrm{kDa}$ and an isoelectric point of 8.49 (GenBank accession number KM192158). The sequence around the first ATG was in good agreement with Kozak's consensus sequence, in that the $n t-3$ position was the A in AAACATG. The putative poly(A) signal (AAGAAA) was observed $35 \mathrm{bp}$ downstream of the translational stop codon.

Sequence analysis of the deduced CpBCK1 revealed a conserved C-terminal catalytic domain of Ser/Thr protein kinase of PKc like superfamily between amino acids 1,328 and 1,598, which has the highest similarity to other fungal BCK1-like proteins of MEKKs (MAP/extracellular signal-regulated kinase kinases) (Supplementary Fig. S1A). Homology searches using the deduced amino acid sequence revealed that the protein product of the cloned $C p B c k 1$ exhibited high similarities with members of the BCK1 homologs of Aspergillus clavatus (36\%), A. flavus (36\% identity), Magnaporthe oryzae (46\%), Saccharomyces cerevisiae (20\%), Trichophyton verrucosum (36\%), and Uncinocarpus reesii (38\%). In addition, the similarity of the deduced sequences of conserved catalytic domains was further increased, i.e., A. clavatus (79\% identity), A. flavus (77\%), Magnaporthe grisea (88\%), Saccharomyces cerevisiae (54\%), Trichophyton verrucosum (78\%), and Uncinocarpus reesii (79\%) (Henikoff and Henikoff 1992). Phylogenetic analysis including homologs representing the pheromone response pathway (Fus3/Kss1) and the osmoregulation/stress response pathway $(\operatorname{Hog} 1)$ indicated that the cloned $\mathrm{CpBCK} 1$ belonged to the BCK1-related group of MAPKKKs.

\section{Construction of the CpBck1-null mutant (TdBCK1).}

To examine the biological functions of the $C p B c k l$ gene, the $C p B c k 1$-null mutant was constructed using site-directed recombination during integrative transformation. Linear DNA containing the disrupted $C p B c k 1$ gene with the $1.4-\mathrm{kb} 5^{\prime}$ and $0.9-\mathrm{kb} 3^{\prime}$ flanking regions was used to transform the virus-free $C$. parasitica EP155/2 strain. A total of 100 stable single-spored transformants were screened by PCR, using the inner hph (hygromycin phosphotransferase gene) and outer (5' distal) gene-specific primers, which corresponded to nt 446 to 465 and -567 to -546 , respectively (relative to the start codon of $h p h$ and $C p B c k l$, respectively). A transformant (TdBCK1) showed the PCR amplicon with the expected size of $3.1 \mathrm{~kb}$, corresponding to the disrupted alleles of the CpBckl gene. To confirm the pure replacement of the wild-type allele with the disrupted allele, the putative null mutant was further examined using Southern blot analysis (Supplementary Fig. S2).
The hybridization pattern of ClaI-digested genomic DNA from the putative $C p B c k 1$-null mutant, with the probe prepared using the 1.1-kb XbaI-digested $C p B c k 1$ fragment, differed from that of the wild-type at $3.1 \mathrm{~kb}$, suggesting that the transforming vector had been integrated at the $C p B c k l$ locus through site-directed homologous recombination. Moreover, the ClaI-digested genomic DNA of transformant showed the hybridizing band at $3.9 \mathrm{~kb}$, corresponding to the expected size of a replaced allele, and the corresponding band also hybridized to the 0.4-kb SalI/BamHI-hph fragment, indicating that the $C p B c k 1$ gene was replaced with part of the transforming vector, as expected.

\section{Phenotypic changes in the TdBCK1 strain.}

The TdBCK1 strain exhibited severely altered colony morphology. TdBCK1 produced abnormal colonies with irregular margins that ceased growth before the colonies reached the edge of the plate. Compared with the wild-type EP155/2 strain, TdBCK1 displayed severely retarded growth with thinner invasive feeding hyphae, near-absence of the typical mycelial mat and aerial hyphae on the surface, and absence of typical spore-forming structures. The TdBCK1 showed diffused pigmentation. However, the pigmentation turned into dark brown rather than the canonical bright yellow as the culture was prolonged (Fig. 1A).

Most interestingly, TdBCK1 began to show sectorization due to the abrupt shortened aerial hyphal growth as culture was prolonged, i.e., usually longer than 7 days. Multiple, usually three to ten, distinctive origins of sectorization were observed on a cultured plate. The origins of sectoring were distributed all over the plate (i.e., not only from the center but also from the margin of the colony). But not all of the mycelia regions became sectored. To determine whether culture conditions affected the sporadic sectorization, we cultured TdBCK1 on different media, such as minimal media and malt extract media, and at two different temperatures, such as 30 and $20^{\circ} \mathrm{C}$ instead of $25^{\circ} \mathrm{C}$. No difference in sectorization regarding the first emergence and frequency of sectors was observed compared with that under the standard growth condition of PDA supplemented with $100 \mathrm{mg}$ of L-methionine and $1 \mathrm{mg}$ of biotin per liter (PDAmb) at $25^{\circ} \mathrm{C}$ (data not shown). When the sectored mycelia were transferred onto a new plate, the sector characteristics were maintained (i.e., active mycelia growth similar to that of the wild type but without developments such as spore production) (Fig. 1B), and no separate group of sectored progeny with different culture morphology was observed.

Complementation of TdBCK1 and sectored progeny of the CpBck1-null mutant (TdBCK1-S1) was conducted using a wild-type $C p B c k 1$ gene. The complemented strains restored the characteristics of asexual sporulation and pigmentation (Fig. 1A). PCR analyses revealed that all of the complemented transformants contained an additional wild-type allele of $C p B c k 1$. Thus, the functional complementation using a wild-type $C p B c k 1$ gene unequivocally confirmed that the phenotypic changes were due to disruption of $C p B c k l$.

In addition to colony morphology, scanning electron microscopic observation was performed (Fig. 2A). Compared with the wild type, TdBCK1 showed a decrease in hyphae elongation and formation of catenulated hypertrophied bulbous or globose cells, indicative of failure to form appropriate cell-wall layers. In contrast, TdBCK1-S1 showed normal hyphae elongation but more dense hyphal distribution than the wild type, as represented by the fluffy growth pattern of sectored mycelia. Compared with hyphae of the original TdBCK1, which showed hyphal swelling as well as atypical branches emerging below the swellings, the hyphae of the sector showed more compact mycelial distribution with numerous aggregations of individual hyphae.

In addition to cellular hypertrophy, transmission electron microscopy (TEM) revealed striking invaginated cell-wall 
growth in the hypertrophied globose or bulbous cells of TdBCK1 (Fig. 2B). Furthermore, the proliferation of new intracellular hyphae, termed intrahyphal hyphae or cell-within-acell (Becker et al. 2015; Bowman et al. 2006; Chen et al. 2014; Kim et al. 2004; Martín-Urdíroz et al. 2008), was observed in TdBCK1, whereas this unusual cellular morphology was not found in wild-type hyphal cells nor the sectored TdBCK1 (Fig. 2B). Close microscopic examination of the hypertrophied globose or bulbous cells by differential interference contrast microscopy and TEM did not show any evidence of degeneration in the outer cells. In addition, live-cell specific fluorescent micrographs using FM4-64 (Hickey et al. 2004) showed fluorescence at the outer cells. These results suggested that the outer cells were still alive (Supplementary Fig. S3). Considering the fact that the TdBCK1 showed morphological defects in cell-wall integrity, we examined the effect of cell wall-perturbing agents on the growth of this mutant. Strains with the same $C p B c k 1$ mutant background, such as TdBCK1 and TdBCK1-S1, showed more severe growth defects on the plates containing cell walldisturbing agents (Fig. 3), indicating that the cloned CpBckl gene is responsible for cell-wall integrity. In addition, susceptibility to the cell wall-degrading enzyme was addressed. The number of protoplasts generated by the $C p B c k 1$-null mutant was significantly higher than that of the wild type. An approximately threefold increase in protoplast numbers from the CpBck1-null mutant was observed, indicating a defect in cellwall integrity (Supplementary Fig. S4).

\section{Virulence assay.}

TdBCK1 was inoculated on excised chestnut tree bark to examine whether disruption of the $C p B c k 1$ gene affected virulence. TdBCK 1 demonstrated that the size of the necrotic area on excised bark was smaller than that of the wild-type strain EP155/2 and even smaller than that of the hypovirulent UEP1 strain, indicating that TdBCK1 is less virulent than both virusfree EP155/2 and virus-containing hypovirulent UEP1 strains (Fig. 4). In addition, the size of the necrotic area of TdBCK1-S1 was smaller than that of the EP155/2 (Fig. 4). Pathogenicity tests using excised bark are strongly correlated with growth defects. Considering that TdBCK1 showed severe growth defects, we expected that it would also show impaired virulence. However, the fact that TdBCK1-S1 showed impaired pathogenicity suggested that growth defects are not the only cause of impaired virulence but that the $C p B c k l$ gene regulates other factors to determine the pathogenicity of this fungus.

\section{DISCUSSION}

A fundamental property of living organisms is the ability to sense and respond in an appropriate manner to changing environmental conditions and various other stimuli. The MAPK pathway is frequently involved in such responses. In fungal pathogens, three representative MAPK pathways (i.e., the pheromone response, cellwall integrity, and osmoregulation/stress response pathways) have been suggested (Zhao et al. 2007). Among these three
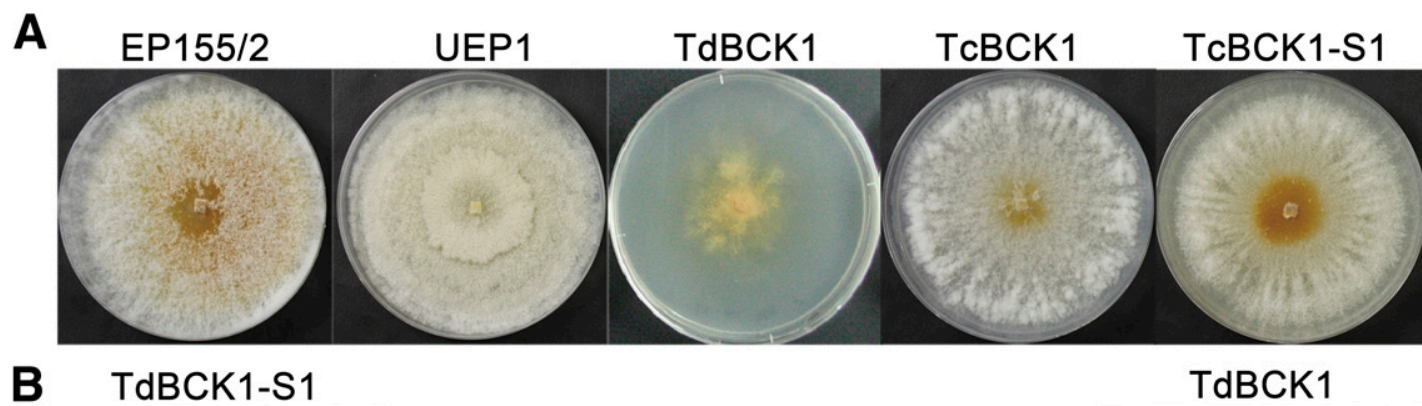

TdBCK1

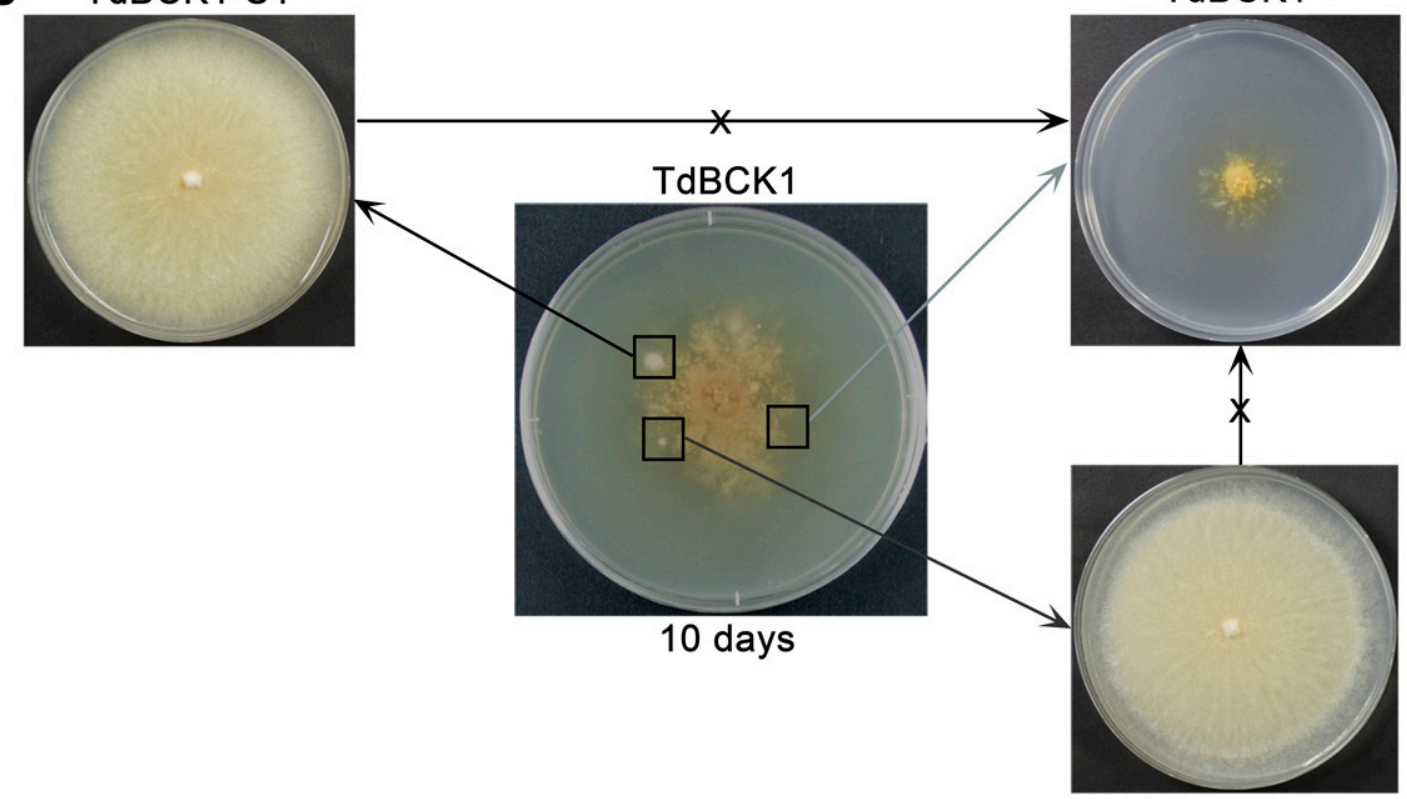

TdBCK1-S2

Fig. 1. Colony morphology of the $C p B c k 1$-null mutant (TdBCK1) strain. A, Strains indicated on the panels are the wild-type EP155/2, hypovirulent UEP1, TdBCK1, a complemented strain of TdBCK1 (TcBCK1), and a complemented strain of sectored progeny (TcBCK1-S1). Colonies after 7 days of culturing are shown. B, A colony of TdBCK1 after 10 days of culturing showed sporadic appearance of sectorization. Arrows indicate colonies of the corresponding regions after 7 days of successive culturing. Note that the sectored phenotype maintained in the successive cultures and the nonsectored area showed the characteristic sick phenotype with sporadic initiation of sectorization. 
pathways, the cell wall-integrity pathway is known to be important in differentiation and pathogenesis. However, studies on the molecular characterization of the involvement of the MAPK pathway in cell-wall integrity are limited in filamentous fungi.

In $C$. parasitca, although $C p k k 1$, a homolog of the MAPKK in the cell wall-integrity pathway, has been suggested to play a role in hypoviral regulation and virulence (Moretti et al. 2014; Rostagno et al. 2010; Turina et al. 2006), its upstream and downstream components have not been characterized. In addition, recent studies of the components of the mating-responsive MAPK pathway reported pathway-related as well as componentspecific phenotypic changes. These data were suggestive of branched signal transduction pathways in this fungus (Park et al. 2012), which prompted us to study $C p B c k l$, a Bckl ortholog of $C$. parasitica.

Analysis of the deduced amino acid sequence of the $C p B c k 1$ gene showed the presence of highly conserved N-terminal regulatory and C-terminal Ser/Thr protein kinase domains, which are hallmark features of this class of enzyme. In addition, it showed high homology to known cell-wall integrity MAPKKKs such as yeast $B c k 1$, suggesting that the cloned $C p B c k 1$ gene is involved in the cell wall-integrity MAPKKK pathway. Phenotypic changes in the $C p B c k 1$-null mutant, including invasive colonial growth, hypersensitivity to cell wall-inhibiting agents, diffused pigmentation, and cell-wall defects observed using electron microscopy confirmed that the cloned gene was indeed the cell wall-integrity MAPKKK.

In addition to cell wall-associated phenotypic changes, TdBCK1 showed very sick colony morphology, including no sign of developmental transition such as conidiation. Lack of aerial mycelia, hypersensitivity to cell-wall inhibitors, and impaired conidiation were also observed in mutant phenotypes of Bckl orthologs, including Coniothyrium minitans (Zeng et al. 2012), A. nidulans (Katayama et al. 2012), and Magnaporthe oryzae (Jeon et al. 2008). However, the progressive autolysis that occurs in other fungi was not observed in $C$. parasitica, suggesting a different level of regulation of cell-wall integrity (i.e., abnormal integrity prohibits the mycelium from further differentiating into aerial hyphae) that terminates autolysis or simply affects the composition of the cell wall, preventing it from being autolyzed. In terms of asexual conidiation, the loss-of-function mutant of CpSte11, a recently characterized
A

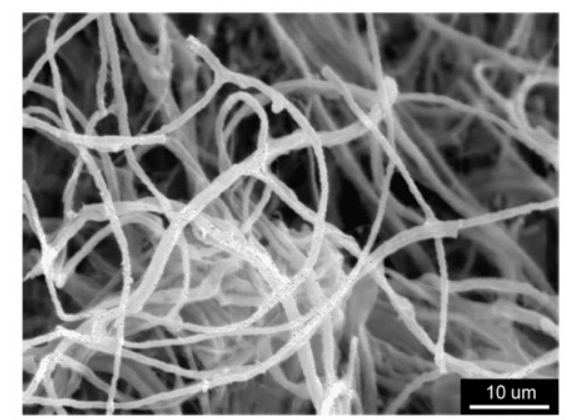

B

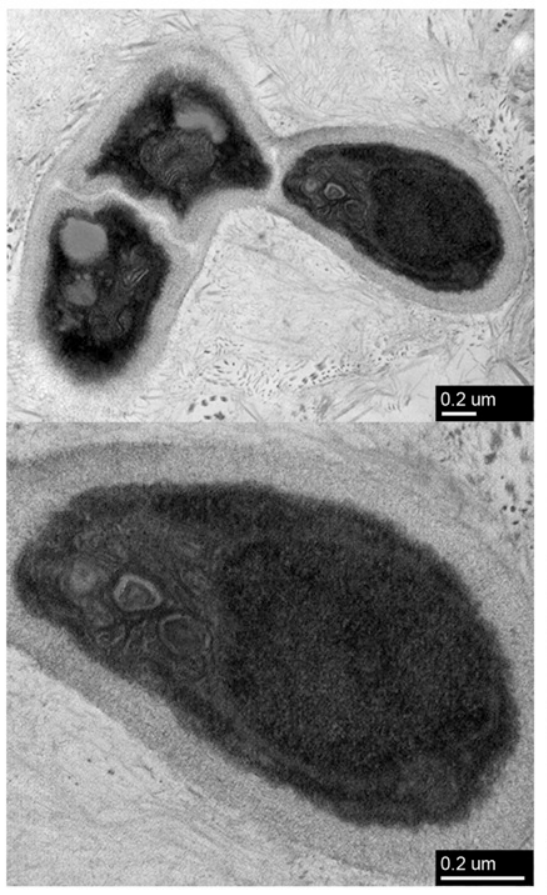

TdBCK1
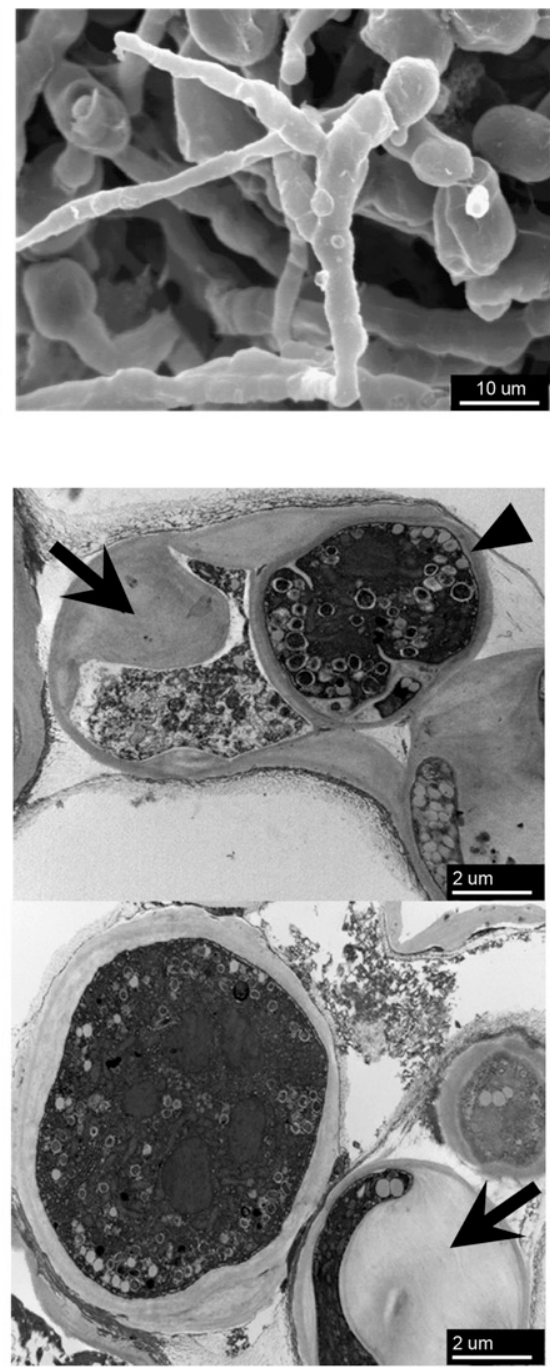

TdBCK1-S1
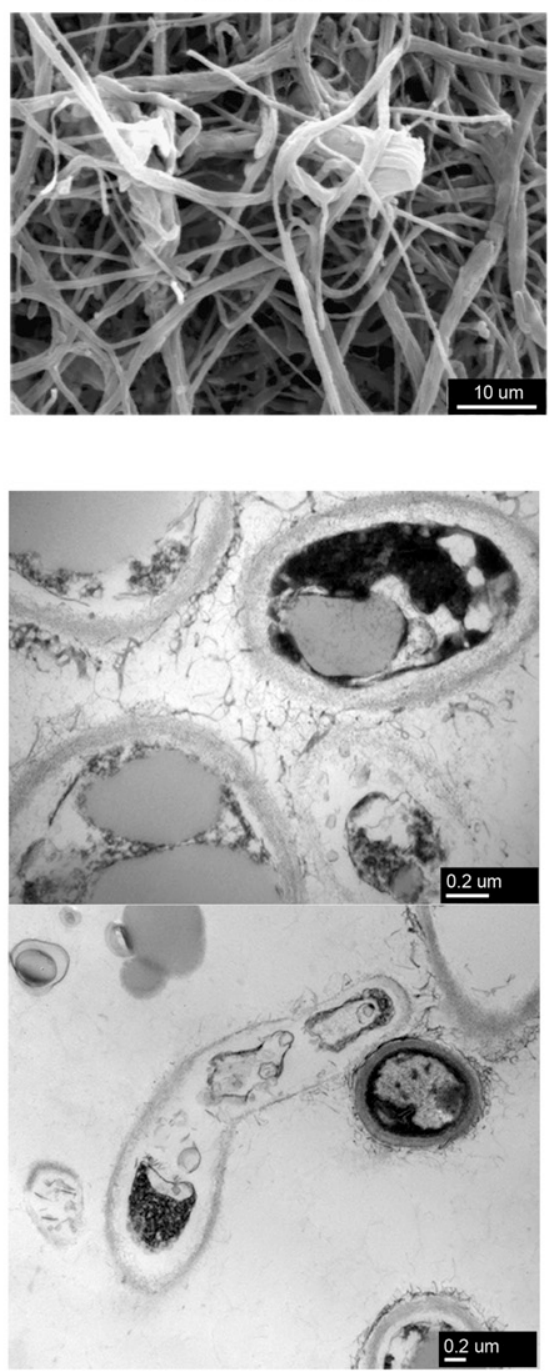

Fig. 2. Ultrastructural characteristics of the $C p B c k 1$-null mutant strain. A, Scanning electron micrographs of mycelia. Typical extended filamentous hyphae are observed in the wild-type EP155/2. Bead-like catenulate hyphae consisting of districted bulbous cells and terminal extension hyphae are seen in the TdBCK1. More dense and thinner individual hyphae with coalesced mycelia are observed in the sectored TdBCK1 (TdBCK1-S1). B, Transmission electron micrographs of mycelia. Typical extended filamentous hyphae are seen in the wild-type EP155/2. Hypertrophied invaginated walls and intrahyphal hyphae are indicated by arrows and arrowheads, respectively, in TdBCK1. Hyphae with less electron-dense material are noted in the TdBCK1-S1. 
MAPKKK ortholog of yeast Ste11, showed enhanced asexual conidiation. However, mutations in the putative downstream components of CpSte 11 showed different phenotypic changes, which suggested that MAPK pathways in $C$. parasitica may have branched. Phenotypic changes in the mutant at the putative downstream components of $C p B c k l$ and $C p k k l$ included many common phenotypes, such as impaired mycelial growth without aerial hyphae, hypersensitivity to cell wall-inhibiting agents, cellwall defects, impaired conidiation, and loss of virulence. However, there were some differences in the mutant phenotypes between the CpBckl-null and Cpkk1-null mutants. Compared with the diffused pigmentation of the $C p B c k l$-null mutant, no pigmentation was observed in the Cpkkl-null mutant. Furthermore, ultrastructural characteristics of TdBCK1 showing invaginated cell-wall growth in the hypertrophied globose or bulbous cells and hyphae grown in hyphae (Becker et al. 2015; Kim et al. 2004; Martín-Urdíroz et al. 2008) were not reported in the Cpkkl-null mutant. These differences in phenotypic changes due to mutations of the genes in the same pathway strongly suggested that the cell wall-integrity MAPK pathway in $C$. parasitica became branched. Hyphae grown in hyphae, which were observed in the globose or bulbous cells, are mainly found in survival forms of fungi, induced by stressful conditions, or various genetic mutations (Brown and Wyllie 1970; Bowman et al. 2006; Chen et al. 2014; Cromey and Cole 1985; Lim et al. 1983; Shankar et al. 1998). These unusual cellular morphologies were further differentiated into
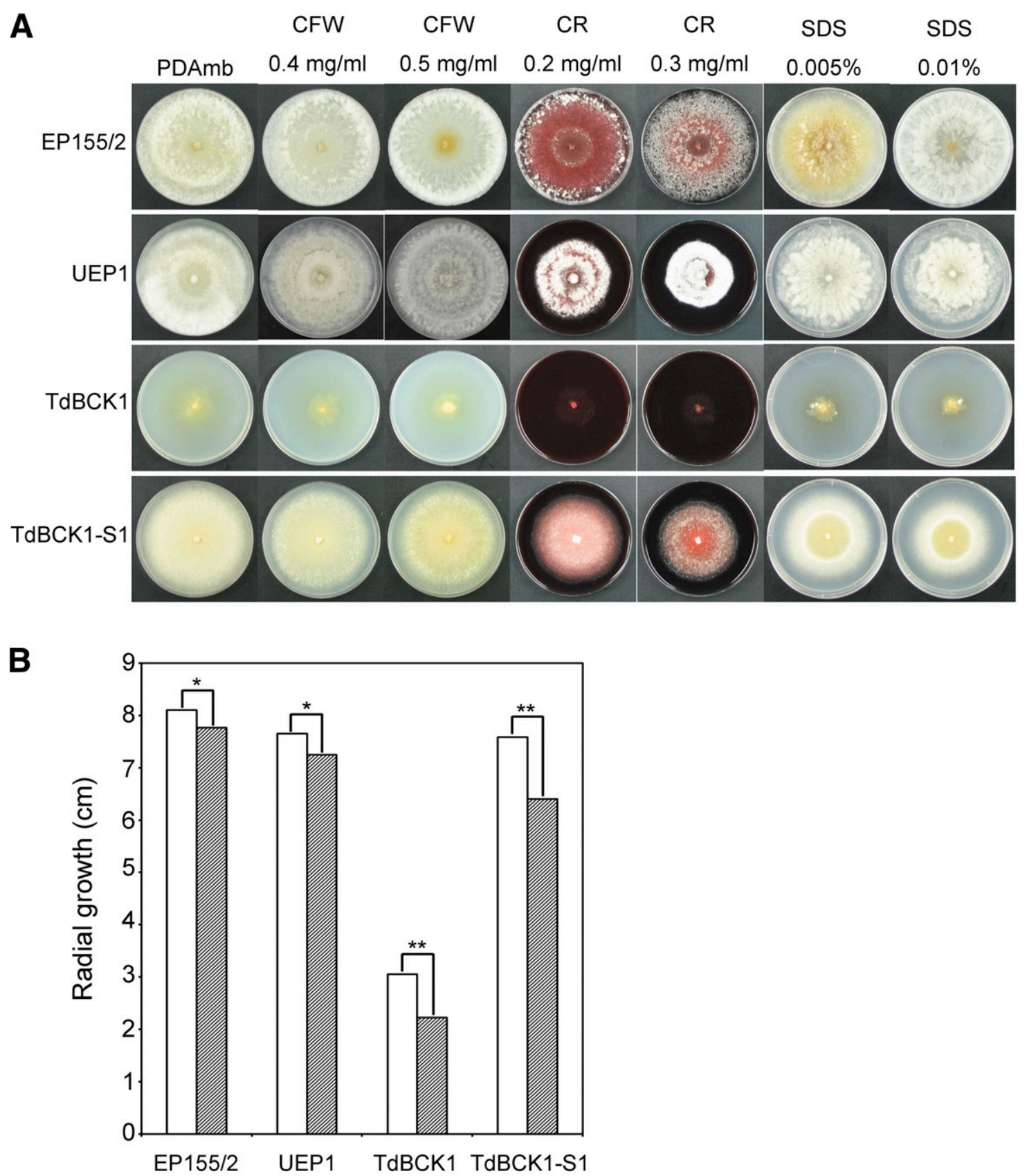

Fig. 3. Effect of various cell wall-perturbing agents on fungal growth. A, The radial growth of the same $C p B c k 1$ mutant background, such as TdBCK1 and TdBCK1-S1, showed more severe growth defects on the plates containing cell wall-disturbing agents. $\mathrm{CFW}=\mathrm{Calcofluor}$ white, $\mathrm{CR}=\mathrm{Congo}$ red, and SDS $=$ sodium dodecyl sulfate. B, Radial growth on plates was assessed every day by measuring the diameter of the colonies that originated from an initial agar plug ( $5 \mathrm{~mm}$ diameter) taken from the edge of an actively growing colony. Six replicates for each strain were used and each experiment was repeated twice. Representative data of colonies cultured for 7 days are shown. Asterisks represent significant differences between the absence (open bar) and presence (solid bar) of the cell wall-disturbing agent CFW $(0.4 \mathrm{mg} / \mathrm{ml})$ at the level of $5 \%(*)$ and $0.1 \%(* *)$. 
'intrahyphal hyphae' and 'cell-within-a-cell' classifications (Bowman et al. 2006). Intrahyphal hyphae are thought to reside within the remnants of older, empty, or degenerating host hyphae. However, cell-within-a-cell organization did not show any evidence indicating that either the inner or the outer cells were degenerating (Bowman et al. 2006). In our microscopic studies of enlarged globose or bulbous cells, many of them exhibited live-cell specific fluorescence at the outer cells, and TEM analysis revealed the presence of electron-dense materials within the outer cells. These results indicated that the outer cells of hyphae grown in hyphae appeared to be metabolically alive or maintained viability during the formation of an inner cell. Thus, the unusual cellular morphology in swollen globose or bulbous hyphae of TdBCK1 has more similarity with the previously described phenomenon of cell-within-a-cell organization. Intrahyphal hyphae formation due to a genetic defect was first observed in the $\operatorname{csm} A$ mutant of A. nidulans (Horiuchi et al. 1999) and was also observed in Epichloëfestucae, which had mutations in the cell wall-integrity MAPK pathway (Becker et al. 2015). In addition, hyphae grown in hyphae were also observed in the novel kinase cppk 1 mutant of $C$. parasitica showing microcolonies (Kim et al. 2004). Therefore, it is interesting to see the relationship between $C p B c k 1$ and $c p p k 1$. It is also noteworthy that MAPKKKs in the two most important MAPK pathways in growth and differentiation processes have opposite effects on the same developmental process (i.e., conidiation).

Most strikingly, TdBCK1 showed sectorization, characterized by fluffy mycelial-type growth compared with the TdBCK1. Because the cell wall-integrity MAPK pathway is a wellconserved MAPK pathway, sectorization could be ascribed as suppressor mutations that may occur at different downstream targets. Therefore, we cloned and sequenced putative downstream targets of $C p B c k 1$, such as orthologs of Slt2 (a cell wall-integrity MAPK, 1,656 bp from 3,240,100 to 3,241,755 of scaffold 7 in the draft genome sequence [MycoCosm Cryphonectria parasitica archive page]), and Swi6 (a downstream transcription factor, $2,866 \mathrm{bp}$ from 278,211 to 281,076 of scaffold 3 in the draft genome sequence). Four different colonies of TdBCK1 and TdBCK1-S1 strains were analyzed for the sequence comparison. However, no differences in the sequences among the wild type, TdBCK1, and TdBCK1-S1 strains were observed (data not shown). In addition, the facts that multiple origins of sectorization were observed on a plate and sporadic sectorization was consistently observed from any parental mutant phenotypic progeny made suppressor mutation unlikely. Many filamentous fungi undergo colony degeneration, producing sectors of fluffy mycelial-type growth without sporulation, which is usually irreversible and inheritable and is often associated with aging (Horgen et al. 1996; Li et al. 1994; Ryan et al. 2003; Wang and St. Leger 2005). Other than the reports on the spontaneous degeneration process in many fungi, little information is available to explain fungal culture instability at the molecular level. However, transcriptional upregulation of MAPKK has been observed in sectored regions of Metarhizium anisopliae (Wang and St. Leger 2005). Moreover, DNA modification such as mitochondrial DNA glycation is known to be associated with sectorization in Metarhizium anisopliae (Li et al. 2008). Although morphological instability of $C$. parasitica has been ascribed to hypovirus infection, it is uncommon in virus-free $C$. parasitica. Therefore, the current study on the regular sectorization from the mutant (not the wild type) indicated that the mutated gene is responsible for sectorization, which appears to be irreversible and inheritable.

Although few studies have explored the relationship between signal transduction pathways and heredity, there is a wellknown case of non-Mendelian heredity generated by the MAPK pathway in Podospora anserina (Kicka and Silar 2004; Kicka et al. 2006). In Podospora anserina, the PaMpk1 MAPK pathway can generate a cytoplasmic and infectious prion-like $C$ element, which results in crippled growth cell degeneration characterized by slower mycelium growth, higher accumulation of pigment, and female sterility. Our observations that mutations in the wellknown conserved component $C p B c k l$ of the signal transduction pathway resulted in the phenotypic change of sectoring and that, once sectored, sectored progenies were stably inherited, suggesting a link between cell wall-integrity signaling and the genetic changes of sectorization. However, the network that connects genetic modifications and signaling may be complex.

Considering that several days were required for development of the sectored phenotype in the parental TdBCK1 strain and not all mycelia regions became sectored, the genetic changes underlying the sectored phenotype appeared to be an adaptive response of the $C p B c k l$-null genotype. Additionally, once sectored, stable maintenance of sectored phenotypes indicated that these adaptive genetic changes were stably inheritable for

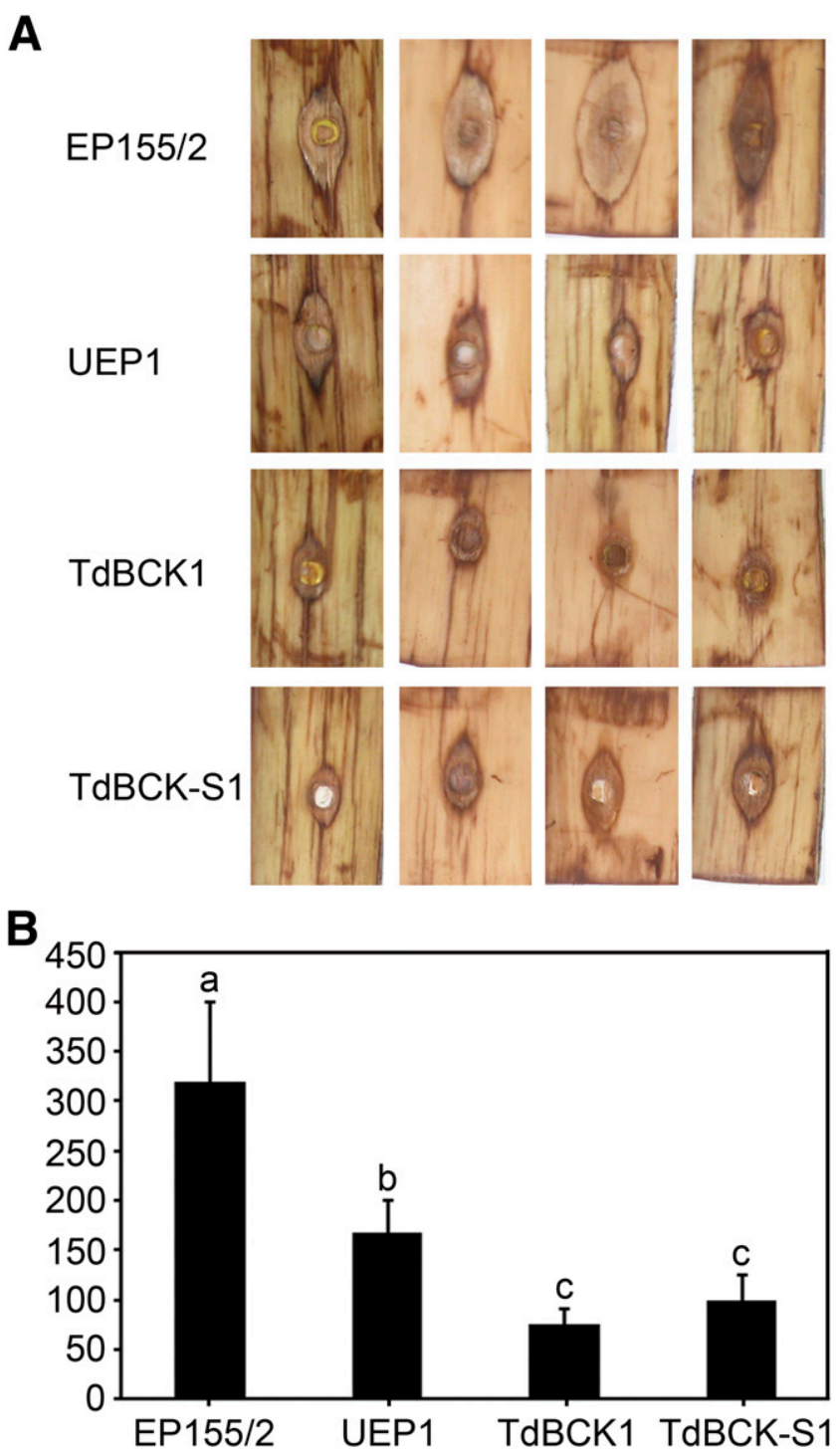

Fig. 4. Virulence assay. A, A representative figure of excised tree bark 1 week after inoculation is shown. The strains used were the virus-free wildtype (EP155/2), its isogenic virus-containing hypovirulent strain (UEP1), TdBCK1, and TdBCK1-S1. B, The lesion measurement values are shown as means \pm standard deviation (square millimeter). Four replicates for each strain were used, and each experiment was repeated three times. Data followed by the same letters above the bars are not significantly different between treatments, according to Duncan's multiple range test at $P=0.05$. 
multiple generations (Cubas et al. 1999). There are several possible explanations for this phenomenon. These include activation of transposable elements. Since the transposable element Crypt1 (GenBank accession number AF283502) is present in the C. parasitica genome, the transposition of Crypt1 was assessed, using Southern blot analysis. As expected, seven copies of the Cryptl gene were present (Supplementary Fig. S5) and no changes in the Cryptl hybridization pattern were observed among the strains. These results indicated that the phenotypic change in sectorization was not due to the transposition of Cryptl. Although the transposable element Crypt1 did not explain the sporadic sectorization and stable sectored phenotype, studies focusing on other explanations, such as epigenetic changes, are warranted.

Our data demonstrated that, in addition to cell-wall-associated phenotypic changes, the mutation of a gene encoding a component of a signal transduction pathway related to cell-wall integrity resulted in sporadic sectorization and these sectored phenotypes were stably inherited. Although the molecular basis of sectorization remains unclear, these data are suggestive of a role for the signal transduction pathway in the regulation of an adaptive genetic modification. Identification of factors related to the genetic functions of $C p B c k l$ will increase our understanding of the regulatory role of $C p B c k 1$ in genetic changes at the molecular level. Moreover, further comprehensive analysis will enable elucidation of the links between phenotypic changes (sectorization) and specific genetic differences.

\section{MATERIALS AND METHODS}

\section{Fungal strains and growth.}

The C. parasitica EP155/2 (American Type Culture Collection 38755) and its isogenic CHV1-713-containing hypovirulent strain UEP1 were maintained on PDAmb plates under constant low light at $25^{\circ} \mathrm{C}$ (Kim et al. 1995). The culture conditions and methods for preparation of the primary inoculum for liquid cultures have been described previously (Kim et al. 1995). The mycelia were collected and lyophilized as described previously until use (Powell and Van Alfen 1987).

\section{Cloning and characterization \\ of a cell wall-integrity MAPKKK gene, $C p B c k 1$.}

The genome database of $C$. parasitica was screened for the $B c k 1$ homolog and PCR amplification was performed using the primers $C p B c k 1$-gF1 (forward) 5'-CGCGTCGCAGCAGATC GTATT-3' and $C p B c k 1$-gR1 (reverse) 5'-CGCCTTGTGAG CGTGGTTTCATT-3'. The resulting 8.9-kb PCR amplicon was cloned into the pGEM-T Easy vector (Promega, Madison, WI, U.S.A.) and was sequenced using the dideoxynucleotide method, with universal and synthetic oligonucleotide primers.

To obtain the cDNA clone of $C p B c k 1$, RT-PCR was performed with primers $C p B c k l-c F 1$ (forward) 5'-GCAACCCAAACAT GTATG-3' and $C p B c k 1$-cR1 (reverse) 5'-TCTCACTTGGTGG TGTTC-3'. The resulting 4.9-kb cDNA amplicon was cloned and sequenced.

\section{Southern blot analysis.}

Genomic DNA from C. parasitica was extracted as described previously (Churchill et al. 1990). DNA $(10 \mu \mathrm{g})$ was digested with the appropriate restriction enzyme, was blotted onto the nylon membrane, and was hybridized with radioactive-labeled probes. To perform transposon analysis, enzyme and probe combinations were used as described previously (Linder-Basso et al. 2001).

\section{Construction of a replacement vector and fungal transformation.}

The replacement vector $\mathrm{pDBCK} 1$, which was designed to favor double-crossover integration events, was constructed as follows. A 6.2-kb XbaI fragment containing the near full-length CpBckl was ligated into SalI-inactivated pBluescriptII SK(+) (Stratagene, La Jolla, CA, U.S.A.), and the resulting plasmid was used as template for inverted PCR, using the primers $5^{\prime}$ GGGTCGACTGTCTTCTGGATGGCGC-3' and 5'-CCGTCGAC TTGACGATACTGCGTAT-3', which incorporate the restriction site for SalI (underlined). The PCR amplicon was digested with SaII and was religated. The resulting plasmid was further digested with SalI and was fused with the 2.4-kb SalI fragment of pDH25, containing the $h p h$ cassette. In the replacement vector pDM3K1, the $h p h$ cassette was inserted between sites 963 and 4,846 bp of the CpBckl gene, relative to the start codon, and was flanked by approximately 1.4 and $0.9 \mathrm{~kb}$ of $5^{\prime}$ and $3^{\prime}$ sequences, respectively. The 4.8-kb SmaI/NotI fragment of pDBCK1 was then used to transform the virus-free EP155/2 strain.

Functional complementation of the $C p B c k 1$-null mutant using a wild-type allele was performed. The complementing vector pCBCK1 was constructed by inserting ApaI-digested pCBCK1 carrying an 8.9-kb fragment containing the full-length $C p B c k 1$ gene into ApaI-digested pBSBE1, which was constructed by inserting a 2.6-kb blunt-ended SalI fragment of pSV50 containing the benomyl resistance cassette (Orbach et al. 1986). The resulting vector was then used to transform the $C p B c k 1$-null mutant.

Protoplast preparation and transformation were performed as described previously (Churchill et al. 1990; Kim et al. 2002; Park et al. 2004). Transformants were selected from agar plates supplemented with $150 \mu \mathrm{g}$ of hygromycin B per milliliter (Calbiochem, San Diego, CA, U.S.A.) or $1.5 \mu \mathrm{g}$ of benomyl per milliliter (DuPont, Wilmington, DE, U.S.A.), passaged three to four times on selective media and single-spore isolated, as described previously (Kim et al. 2002). PCR and Southern blot analysis was conducted with genomic DNA from the transformants, to confirm the replacement and in trans complementation of the $C p B c k 1$ gene.

\section{Characteristics of the $C p B c k 1$-null mutant.}

The phenotypic and molecular characteristics of the CpBck1null mutant were compared with wild-type EP155/2 and the hypovirulent UEP1 strains. Phenotypic changes in growth rate, pigmentation, conidiation, and mating capability were measured as described previously (Kim et al. 2002; Powell and Van Alfen 1987). Morphological characteristics were investigated on PDAmb medium with cell wall-perturbing agents such as calcoflour white, Congo red, or sodium dodecyl sulfate (Valiante et al. 2009). A virulence test using excised chestnut tree bark was conducted according to Lee et al. (1992).

To test the sensitivity of the $C p B c k 1$-null mutant to cell wall-degrading enzymes, protoplast preparation was performed as described previously (Churchill et al. 1990; Kim et al. 2002; Park et al. 2004). One gram of harvested mycelium was digested in $10 \mathrm{ml}$ of osmotically stabilized enzyme solution. After $4 \mathrm{~h}$ of digestion, the concentration of protoplasts was measured using a hemacytometer and the parental wild-type EP155/2 was used as a control.

\section{Fluorescent microscopy.}

To evaluate the viability of hyphae of the $C$. parasitica strains, mycelial fragments were stained with $5 \mu \mathrm{g}$ of FM4-64 per milliliter (Life Technology, Eugene, OR, U.S.A.) on ice for $30 \mathrm{~min}$. After the hyphae were washed three times with phosphate buffered saline by centrifugation at $3,000 \times g$ for $5 \mathrm{~min}$ at room temperature, the cells were examined using a confocal laser scanning microscope (LSM 510 META model, Carl Zeiss, Oberkochen, Germany).

\section{Scanning electron microscopy (SEM) and TEM.}

To evaluate the cell-wall integrity of hyphae of the C. parasitica strains under SEM and TEM, fungal mycelia were 
cultured for 10 days on PDAmb. Briefly, mycelial fragments detached from the outermost region of fungal colonies were fixed with Karnovsky's fixative in $0.05 \mathrm{M}$ cacodylate buffer at $\mathrm{pH} 7.2$ (Karnovsky 1965). After postfixation with $1 \% \mathrm{OsO}_{4}$ in $0.05 \mathrm{M}$ cacodylate buffer at $\mathrm{pH} 7.2$ and dehydration through a graded ethanol series of $30,50,70,80,90$, and $100 \%$ for $10 \mathrm{~min}$ each, the specimens were coated with gold, using a Sputter Coater (JFC-1100E, JEOL, Tokyo). The specimens were observed under a JSM-5410LV scanning electron microscope (JEOL) at $20 \mathrm{kV}$ (Kim et al. 2004).

After the dehydration of mycelia stage, fungal samples were embedded in Spurr's epoxy resin following the standard procedures for specimen preparation for TEM (Kim et al. 2001, 2004). Semithin 1- $\mu$ m-thick sections made with a glass knife were used for light microscopy after staining with $1 \%$ toluidine blue O. Ultrathin 80- to 90-nm-thick sections of embedded samples were cut with a diamond knife, were stained with uranyl acetate and lead citrate, and were observed using a JEOL transmission electron microscope (JEM-1010, JEOL) at $80 \mathrm{kV}$.

\section{Statistical analysis.}

Colony diameters and canker areas were analyzed by analysis of variance using SPSS version 15 (SPSS, Chicago) and the significance of the effects of the strains and treatments was determined, using Duncan's multiple range test at $P=0.05$ and $P=0.001$

\section{ACKNOWLEDGMENTS}

This work was supported by National Research Foundation of Korea (NRF) grants funded by Korea's Ministry of Science (MSIP) (numbers 2015R1A2A1A10055684 and 2012R1A1A3015582). This research was also supported in part by the Korea Research Council of Fundamental Science \& Technology (Joint Degree and Research Center for Biorefinery). We thank the Institute of Molecular Biology and Genetics at Chonbuk National University for kindly providing the facilities for this research. K. K. So and Y. H. Ko were supported by BK21 PLUS program in the Department of Bioactive Material Sciences.

\section{LITERATURE CITED}

Allen, T. D., Dawe, A. L., and Nuss, D. L. 2003. Use of cDNA microarrays to monitor transcriptional responses of the chestnut blight fungus Cryphonectria parasitica to infection by virulence-attenuating hypoviruses. Eukaryot. Cell 2:1253-1265.

Allen, T. D., and Nuss, D. L. 2004. Specific and common alterations in host gene transcript accumulation following infection of the chestnut blight fungus by mild and severe hypoviruses. J. Virol. 78:4145-4155.

Anagnostakis, S. L. 1982. Biological control of chestnut blight. Science 215:466-471.

Becker, Y., Eaton, C. J., Brasell, E., May, K. J., Becker, M., Hassing, B., Cartwright, G. M., Reinhold, L., and Scott, B. 2015. The fungal cell-wall integrity MAPK cascade is crucial for hyphal network formation and maintenance of restrictive growth of Epichloëfestucae in symbiosis with Lolium perenne. Mol. Plant-Microbe Interact 28:69-85.

Bowman, S. M., Piwowar, A., Al Dabbous, M., Vierula, J., and Free, S. J. 2006. Mutational analysis of the glycosylphosphatidylinositol (GPI) anchor pathway demonstrates that GPI-anchored proteins are required for cell wall biogenesis and normal hyphal growth in Neurospora crassa. Eukaryot. Cell 5:587-600.

Brown, M. F., and Wyllie, T. D. 1970. Ultrastructure of microsclerotia of Verticillium albo-atrum. Phytopathology 60:538-542.

Chen, B., Gao, S., Choi, G. H., and Nuss, D. L. 1996. Extensive alteration of fungal gene transcript accumulation and elevation of G-protein-regulated cAMP levels by a virulence-attenuating hypovirus. Proc. Natl. Acad. Sci. U.S.A. 93:7996-8000.

Chen, D., Wang, Y., Zhou, X., Wang, Y., and Xu, J. R. 2014. The Sch9 kinase regulates conidium size, stress responses, and pathogenesis in Fusarium graminearum. PLoS One 9:e105811.

Choi, E. S., Chung, H. J., Kim, M. J., Park, S. M., Cha, B. J., Yang, M. S., and Kim, D. H. 2005. Characterization of the ERK homologue CpMK2 from the chestnut blight fungus Cryphonectria parasitica. Microbiology 151:1349-1358.
Churchill, A. C. L., Ciuffetti, L. M., Hansen, D. R., Vanetten, H. D., and Van Alfen, N. K. 1990. Transformation of the fungal pathogen Cryphonectria parasitica with a variety of heterologous plasmids. Curr. Genet. 17:25-31.

Cromey, M. G., and Cole, A. L. J. 1985. Cytology of the host-pathogen interactions between Lolium perenne and Drechslera dictyoides. Plant Pathol. 34:83-94.

Cubas, P., Vincent, C., and Coen, E. 1999. An epigenetic mutation responsible for natural variation in floral symmetry. Nature 401:157-161.

Deng, F., Allen, T. D., Hillman, B. I., and Nuss, D. L. 2007. Comparative analysis of alterations in host phenotype and transcript accumulation following hypovirus and mycoreovirus infections of the chestnut blight fungus Cryphonectria parasitica. Eukaryot. Cell 6:1286-1298.

Elliston, J. E. 1985. Characteristics of dsRNA-free and dsRNA-containing strains of Endothia parasitica in relation to hypovirulence. Phytopathology 75:151-158.

Gao, S., and Nuss, D. L. 1996. Distinct roles for two G protein alpha subunits in fungal virulence, morphology, and reproduction revealed by targeted gene disruption. Proc. Natl. Acad. Sci. U.S.A. 93:14122-14127.

Havir, E. A., and Anagnostakis, S. L. 1983. Oxalate production by virulent but not by hypovirulent strains of Endothia parasitica. Physiol. Plant Pathol. 23:369-376.

Henikoff, S., and Henikoff, J. G. 1992. Amino acid substitution matrices from protein blocks. Proc. Natl. Acad. Sci. U.S.A. 89:10915-10919.

Herskowitz, I. 1995. MAP kinase pathways in yeast: for mating and more. Cell 80:187-197.

Hickey, C. P., Swift, S. R., Roca, M. G., and Read, N. D. 2004. Live-cell imaging of filamentous fungi using vital fluorescent dyes and confocal microscopy. Methods Microbiol. 34:63-87.

Horgen, P. A., Carvalho, D., Sonnenberg, A., Li, A., and Van Griensven, L. J. L. D. 1996. Chromosomal abnormalities associated with strain degeneration in the cultivated mushroom, Agaricus bisporus. Fungal Genet. Biol. 20:229-241.

Horiuchi, H., Fujiwara, M., Yamashita, S., Ohta, A., and Takagi, M. 1999. Proliferation of intrahyphal hyphae caused by disruption of $\operatorname{csm} A$, which encodes a class $\mathrm{V}$ chitin synthase with a myosin motor-like domain in Aspergillus nidulans. J. Bacteriol. 181:3721-3729.

Jeon, J., Goh, J., Yoo, S., Chi, M. H., Choi, J., Rho, H. S., Park, J., Han, S. S., Kim, B. R., Park, S. Y., Kim, S., and Lee, Y. H. 2008. A putative MAP kinase kinase kinase, MCK1, is required for cell wall integrity and pathogenicity of the rice blast fungus, Magnaporthe oryzae. Mol. PlantMicrobe Interact 21:525-534.

Kang, H. S., Choi, J. W., Park, S. M., Cha, B. J., Yang, M. S., and Kim, D. H. 1999. Ordered differential display from Cryphonectria parasitica. J. Plant Pathol. 16:142-146.

Karnovsky, M. J. 1965. A formaldehyde-glutaraldehyde fixative of high osmolarity for use in electron microscopy. J. Cell Biol. 27:137A.

Kasahara, S., and Nuss, D. L. 1997. Targeted disruption of a fungal G-protein beta subunit gene results in increased vegetative growth but reduced virulence. Mol. Plant-Microbe Interact 10:984-993.

Katayama, T., Uchida, H., Ohta, A., and Horiuchi, H. 2012. Involvement of protein kinase $\mathrm{C}$ in the suppression of apoptosis and in polarity establishment in Aspergillus nidulans under conditions of heat stress. PLoS One 7:e50503.

Kazmierczak, P., Pfeiffer, P., Zhang, L., and Van Alfen, N. K. 1996. Transcriptional repression of specific host genes by the mycovirus Cryphonectria hypovirus 1. J. Virol. 70:1137-1142.

Kicka, S., Bonnet, C., Sobering, A. K., Ganesan, L. P., and Silar, P. 2006. A mitotically inheritable unit containing a MAP kinase module. Proc. Natl. Acad. Sci. U.S.A. 103:13445-13450

Kicka, S., and Silar, P. 2004. PaASK1, a mitogen-activated protein kinase kinase kinase that controls cell degeneration and cell differentiation in Podospora anserina. Genetics 166:1241-1252.

Kim, D. H., Rigling, D., Zhang, L., and Van Alfen, N. K. 1995. A new extracellular laccase of Cryphonectria parasitica is revealed by deletion of Lac1. Mol. Plant-Microbe Interact 8:259-266.

Kim, K. W., Park, E. W., Kim, Y. H., Ahn, K. K., Kim, P. G., and Kim, K. S. 2001. Latency- and defense-related ultrastructural characteristics of apple fruit tissues infected with Botryosphaeria dothidea. Phytopathology 91:165-172.

Kim, M. J., Choi, J. W., Park, S. M., Cha, B. J., Yang, M. S., and Kim, D. H. 2002. Characterization of a fungal protein kinase from Cryphonectria parasitica and its transcriptional upregulation by hypovirus. Mol. Microbiol. 45:933-941.

Kim, M. J., Park, S. M., Kim, Y. H., Cha, B. J., Yang, M. S., and Kim, D. H. 2004. Deletion of a hypoviral-regulated cppk 1 gene in a chestnut blight fungus, Cryphonectria parasitica, results in microcolonies. Fungal Genet. Biol. 41:482-492. 
Lee, J. K., Tattar, T. A., Berman, P. M., and Mount, M. S. 1992. A rapid method for testing the virulence of Cryphonectria parasitica using excised bark and wood of American chestnut. Phytopathology 82:14541456.

Li, A., Begin, M., Kokurewicz, K., Bowden, C., and Horgen, P. A. 1994. Inheritance of strain instability (sectoring) in the commercial button mushroom, Agaricus bisporus. Appl. Environ. Microbiol. 60:23842388.

Li, L., Pischetsrieder, M., St Leger, R. J., and Wang, C. 2008. Associated links among mtDNA glycation, oxidative stress and colony sectorization in Metarhizium anisopliae. Fungal Genet. Biol. 45:1300-1306.

Lim, L. L., Fineran, B. A., and Cole, A. L. J. 1983. Ultrastructure of intrahyphal phypahe of Glomus fasciculatum (Thaxter) Gerdemann and Trappe in roots of white clover (Trifoloium repens L.). New Phytol. 95: 231-239.

Linder-Basso, D., Foglia, R., Zhu, P., and Hillman, B. I. 2001. Crypt1, an active Ac-like transposon from the chestnut blight fungus, Cryphonectria parasitica. Mol. Genet. Genomics 265:730-738.

Martín-Urdíroz, M., Roncero, M. I., González-Reyes, J. A., and RuizRoldán, C. 2008. ChsVb, a class VII chitin synthase involved in septation, is critical for pathogenicity in Fusarium oxysporum. Eukaryot. Cell 7:112-121.

Moretti, M., Rossi, M., Ciuffo, M., and Turina, M. 2014. Functional characterization of the three mitogen-activated protein kinase kinases (MAP2Ks) present in the Cryphonectria parasitica genome reveals the necessity of Cpkk1 and Cpkk2, but not Cpkk3, for pathogenesis on chestnut (Castanea spp.). Mol. Plant Pathol. 15:500-512.

Nuss, D. L. 1992. Biological control of chestnut blight: an example of virusmediated attenuation of fungal pathogenesis. Microbiol. Rev. 56:561-576.

Orbach, M. J., Porro, E. B., and Yanofsky, C. 1986. Cloning and characterization of the gene for beta-tubulin from a benomyl-resistant mutant of Neurospora crassa and its use as a dominant selectable marker. Mol. Cell. Biol. 6:2452-2461.

Park, J. A., Kim, J. M., Park, S. M., and Kim, D. H. 2012. Characterization of CpSte11, a MAPKKK gene of Cryphonectria parasitica, and initial evidence of its involvement in the pheromone response pathway. Mol. Plant Pathol. 13:240-250.

Park, S. M., Choi, E. S., Kim, M. J., Cha, B. J., Yang, M. S., and Kim, D. H. 2004 Characterization of HOG1 homologue, CpMK1, from Cryphonectria parasitica and evidence for hypovirus-mediated perturbation of its phosphorylation in response to hypertonic stress. Mol. Microbiol. 51:1267-1277.

Powell, W. A. J., Jr., and Van Alfen, N. K. 1987. Two nonhomologus viruses of Cryphonectria (Endothia) parasitica reduce accumulation of specific virulence-associated polypeptides. J. Bacteriol. 169:5324-5326.

Rigling, D., Heiniger, U., and Hohl, H. R. 1989. Reduction of laccase activity in dsRNA-containing hypovirulent strains of Cryphonectria (Endothia) parasitica. Phytopathology 79:219-223.

Rostagno, L., Crivelli, G., and Turina, M. 2010. Study of mRNA expression by real time PCR of Cpkk1,Cpkk2 and Cpkk3, three MEKs of Cryphonectria parasitica, in virus-free and virus-infected isogenic isolates. J. Phytopathol. 158:409-416.

Ryan, M. J., Smith, D., Bridge, P. D., and Jeffries, P. 2003. The relationship between fungal preservation method and secondary metabolite production in Metarhizium anisopliae and Fusarium oxysporum. World J. Microbiol. Biotechnol. 19:839-844.

Schaeffer, H. J., and Weber, M. J. 1999. Mitogen-activated protein kinases: Specific messages from ubiquitous messengers. Mol. Cell. Biol. 19:24352444.

Shankar, M., Cowling, W. A., and Sweetingham, M. W. 1998. Histological observations of latent infection and tissue colonization by Diaporthe toxica in resistant and susceptible narrow-leafed lupins. Can. J. Bot. 76: 1305-1316.

Sun, Q., Choi, G. H., and Nuss, D. L. 2009. Hypovirus-responsive transcription factor gene prol of the chestnut blight fungus Cryphonectria parasitica is required for female fertility, asexual spore development, and stable maintenance of hypovirus infection. Eukaryot. Cell 8:262270.

Turina, M., Zhang, L., and Van Alfen, N. K. 2006. Effect of Cryphonectria hypovirus 1 (CHV1) infection on Cpkk1, a mitogen-activated protein kinase kinase of the filamentous fungus Cryphonectria parasitica. Fungal Genet. Biol. 43:764-774.

Valiante, V., Jain, R., Heinekamp, T., and Brakhage, A. A. 2009. The MpkA MAP kinase module regulates cell wall integrity signaling and pyomelanin formation in Aspergillus fumigatus. Fungal Genet. Biol. 46:909-918.

Van Alfen, N. K. 1982. Biology and potential for disease control of hypovirulence of Endothia parasitica. Annu. Rev. Phytopathol. 20 349-362.

Van Alfen, N. K., Jaynes, R. A., Anagnostakis, S. L., and Day, P. R. 1975. Chestnut blight: biological control by transmissible hypovirulence in Endothia parasitica. Science 189:890-891.

Wang, C., and St. Leger, R. J. 2005. Developmental and transcriptional responses to host and nonhost cuticles by the specific locust pathogen Metarhizium anisopliae var. acridum. Eukaryot. Cell 4:937-947.

$\mathrm{Xu}$, J. R. 2000. Map kinases in fungal pathogens. Fungal Genet. Biol. 31: 137-152.

Zeng, F., Gong, X., Hamid, M. I., Fu, Y., Jiatao, X., Cheng, J., Li, G., and Jiang, D. 2012. A fungal cell wall integrity-associated MAP kinase cascade in Coniothyrium minitans is required for conidiation and mycoparasitism. Fungal Genet. Biol. 49:347-357.

Zhao, X., Mehrabi, R., and Xu, J. R. 2007. Mitogen-activated protein kinase pathways and fungal pathogenesis. Eukaryot. Cell 6:1701-1714.

\section{AUTHOR-RECOMMENDED INTERNET RESOURCE}

JGI MycoCosm database Cryphonectria parasitica archive page: http://genome.jgi-psf.org/Crypa1/Crypa1.home.htm 